\title{
PSEUDOEXFOLIATION SYNDROME AND PSEUDOEXFOLIATION GLAUCOMA
}

\author{
M Shiraz Ali' ${ }^{1}$ Rajiv Kumar Gupta²
}

${ }^{1}$ Consultant Ophthalmologist, "Eye N You", Advanced Center for Cataract and Glaucoma Management, Kantatoli, Ranchi, Jharkhand. ${ }^{2}$ Associate Professor, Regional Institute of Ophthalmology, Rajendra Institute of Medical Sciences, Bariatu, Ranchi, Jharkhand.

\section{ABSTRACT}

\section{BACKGROUND}

Pseudoexfoliation syndrome is a systemic disorder characterised by the deposition of white dandruff-like material within the anterior segment of the eye and other organs such as the heart, lungs and kidneys. The deposition of this material in the trabecular meshwork can result in aqueous flow obstruction, raised intraocular pressure and glaucoma. In fact, pseudoexfoliation glaucoma is the most common form of secondary open-angle glaucoma. In contrast to primary open-angle glaucoma, this disease runs a more aggressive clinical course with high intraocular pressure at onset, faster rates of progression, poor response to medical therapy and increased need for surgical intervention.

\section{KEYWORDS}

Pseudoexfoliation Syndrome, Pseudoexfoliation Glaucoma, LOXL1 Gene.

HOW TO CITE THIS ARTICLE: Ali MS, Gupta RK. Pseudoexfoliation syndrome and pseudoexfoliation glaucoma. J. Evolution Med. Dent. Sci. 2017;6(73):5267-5273, DOI: 10.14260/Jemds/2017/1143

\section{BACKGROUND}

Pseudoexfoliation Syndrome (PXFS) was first described by an ophthalmologist from Finland named John Lindberg in 1917. Several decades later, an ocular pathologist named Georgiana Dvorak-Theobald suggested the term "pseudoexfoliation" to distinguish it from a similar ailment, which sometimes affected glass blowers called "true exfoliation syndrome" that was described by Anton Elschnig in 1922. Sometimes, the two terms, "pseudoexfoliation" and "exfoliation" are used interchangeably. PXFS is a disease of basement membrane of tissue lining the posterior chamber of the eye and possibly other sites. Pseudoexfoliation Glaucoma (PXFG) is the most common type of secondary open-angle glaucoma.

\section{Epidemiology}

Prevalence figures show a wide variation between $0.2 \%$ and $27 \%$ for PXFS and between $0.07 \%$ and $14.2 \%$ for PXFG depending on the region. ${ }^{1}$ Geographic and ethnic differences appear to be important with PXFS prevalences worldwide varying from $0 \%$ in Inuit populations in Alaska, Greenland and Canada to $38 \%$ in the Navajo population in the United States. PXFS is also widely prevalent in the Scandinavian countries, Europe, The United Kingdom and The Middle East. ${ }^{2}$ PXFS has been found in East and South Africa, India, Southeast Asia, Australia and many regions in South America.

PXFS is more common in older age groups with most cases occurring in the late $60 \mathrm{~s}$ and early $70 \mathrm{~s}$. There is no clear relation between PXFS prevalence and gender. However, one study from India found that PXFS is more common in females, but males appear to be at greater risk of developing glaucoma

Financial or Other, Competing Interest: None.

Submission 04-08-2017, Peer Review 27-08-2017,

Acceptance 02-09-2017, Published 11-09-2017.

Corresponding Author:

Dr. M. Shiraz Ali,

"Eye N You",

Advanced Center for Cataract and Glaucoma Management,

$3^{\text {rd }}$ Floor, NILE Complex,

Old Hazaribag Road, Kantatoli,

Ranchi - 834001, Jharkhand, India.

E-mail: mshirazali786@gmail.com

DOI: $10.14260 /$ jemds $/ 2017 / 1143$ if they have PXFS. ${ }^{2}$ The condition maybe unilateral or bilateral and over half of unilateral cases become bilateral over a 20-year period. ${ }^{3}$

Overall, studies indicate about $40 \%$ of PXFS patients will develop glaucoma. ${ }^{4}$ The reported prevalence of PXFS among patients with open-angle glaucoma also shows considerable geographic variation with 26\% in Denmark, $75 \%$ in Sweden, $60 \%$ in Norway, $46.9 \%$ in the Mediterranean area of Turkey and $44.5 \%$ in the northwest of Spain compared with reports of $1 \%$ to $12 \%$ in the United States. 5

\section{Genetics and Pathophysiology}

Polymorphisms in the coding region of LOXL1 (lysyl oxidaselike protein 1) located on chromosome 15q24 are associated with PXFS and PXFG. ${ }^{6}$ LOXL1 is one of many enzymes essential for the formation of elastin fibers. It plays a role in modifying tropoelastin, the basic building block of elastin and catalyses the process for monomers to cross-link and form elastin. Additionally, genetic variants of Contactin-Associated Protein-Like 2 (CNTNAP2) and Clusterin (CLU) are being evaluated as potential catalysts for PXFG. ${ }^{7}$

The primary mechanism of glaucomatous damage in PXFS is the impedance of aqueous outflow. The accumulation of Pseudoexfoliative (PEX) material in the Trabecular Meshwork (TM) and subsequent degenerative changes to the tissue pose the greatest resistance to proper drainage. Additionally, it has been shown that active localised production of PEX material by the endothelium of Schlemm's canal can cause lumen narrowing. ${ }^{8}$ Proliferation of PEX material by the corneal endothelial cells as well as secondary migration posteriorly over the TM may further lead to increased Intraocular Pressure (IOP). ${ }^{8}$

\section{Histology and Proteomics}

Ultrastructurally, PEX deposits are electron dense, elastotic material that appear fibrillar in structure. Histochemical studies reported that PEX material consists of a core protein surrounded by glycoconjugates giving it a glycoprotein/proteoglycan structure. Exfoliation aggregates can be seen both intracellularly while being synthesised and extracellularly where they are deposited. 
Proteomic analysis demonstrates that PEX material composition can be categorised into elastic fibers, basement membrane and blood-derived components. ${ }^{9}$ Interestingly, a number of complement factor proteins are also present in PEX deposits. ${ }^{9}$

\section{Ocular and Systemic Sources}

PEX material is produced by many cell types in the anterior segment including lens capsule epithelium, iris epithelium, vascular endothelium, corneal endothelium and Schlemm's canal endothelium. ${ }^{10}$ The material has also been found in extrabulbar tissue including the conjunctiva, extraocular muscles, orbital septa, posterior ciliary arteries, vortex veins and central retinal vessels passing through the optic nerve sheaths. ${ }^{11}$

PEX material has also been demonstrated in tissues throughout the body of patients including lung, heart, liver, gallbladder, skin, kidney and cerebral meninges ${ }^{12}$ suggesting a systemic process involving generalised abnormal elastin metabolism.

A review of the Blue Mountains Eye Study, Australian population data suggested that PXFS was associated with hypertension and any history of vascular events (such as angina, heart attack and stroke). ${ }^{13}$ Plasma homocysteine level is also increased in PXFS and PXFG and homocysteine level increases with the duration of PXFS. ${ }^{14}$ Also, these patients present greater serum concentrations of anti-Helicobacter pylori IgG antibody compared to healthy patients ${ }^{15}$ and an elevation of anti-Helicobacter pylori IgG antibody has been demonstrated in the aqueous humour of PXFG and POAG patients. ${ }^{15}$

\section{Clinical and Pathologic Features Corneal Changes}

Flakes of PEX material and pigment accumulation maybe seen on the corneal endothelium, scattered diffusely or in the form of a vertical spindle similar to the Krukenberg's spindle in pigmentary glaucoma. Specular microscopy of the corneal endothelium has revealed a significantly lower than normal cell density in eyes with PXFS and changes in cell size and shape. ${ }^{16}$ These finding have also been observed in the unaffected eye of unilateral cases. ${ }^{16}$ These changes predispose to early corneal decompensation at only moderate rises of IOP or after cataract surgery. In certain instances, these changes are accompanied by increased central corneal thickness. ${ }^{17}$

\section{Iris Changes}

PEX material may also be seen as white flecks on the pupillary margin of the iris with loss of pigment at the pupillary ruff ${ }^{18}$ [Photo 1]. Iris transillumination typically reveals a moth-eaten pattern near the pupillary sphincter, and many patients also have diffuse mid-peripheral transillumination defects ${ }^{18}$ [Photo 2]. Transillumination of the iris from behind by light applied to the sclera near the equator commonly demonstrates a patchy loss of pigment from the posterior pigment layer adjoining the pupil, but there is no clear indication of loss from peripheral portions of the iris.

Fluorescein angiographic studies of the iris have revealed hypoperfusion, peripupillary leakage and neovascularisation. ${ }^{19}$ Whether a primary or secondary feature, the iris hypoxia is associated with atrophy of the iris pigment epithelium, stroma and muscle cells. ${ }^{20}$ Atrophy of the pigment epithelium maybe associated with anterior chamber melanin dispersion, which may be seen as a whorl-like pattern of pigment particles on the iris sphincter and pigment deposition on the peripheral iris, ${ }^{18}$ whereas atrophy of the muscle cells may account for the poor mydriasis. ${ }^{20}$

\section{Lens, Zonules and Ciliary Body Changes}

In the fully developed form of PXFS, PEX material is visible on the anterior lens surface in a typical three distinct zone- the relatively homogenous central disc corresponds to the size of the undilated pupil. The central disc is surrounded by a clear intermediate zone probably corresponding to the contact with the moving iris. The peripheral zone is a granular, often layered ring of deposits, which may have radial striations. ${ }^{21}$ The central zone is absent in about $20 \%$ of cases or more, but the peripheral defect is a consistent finding and the pupil must be dilated before the lens changes can be seen in some cases [Photo 3].

Cataract occurs frequently in eyes with PXFS. ${ }^{5}$ Cataracts in these eyes have a higher percentage of nuclear opacities and smaller percentage of cortical and supranuclear opacities. ${ }^{22}$ Recent studies have also demonstrated a higher rate of subcapsular cataract in PXFS with respect to non-PXFS eyes. ${ }^{15}$

PEX material may be detected earliest on the ciliary processes and zonules. Involvement of zonules can lead to lens subluxation and phacodonesis. ${ }^{23}$ Proteolytic enzymes in the PEX material may facilitate zonular disintegration.

\section{Gonioscopic Findings}

Pigment accumulation in the angle is pathognomonic of the condition and is seen as a wavy pigment deposition above Schwalbe's line (called Sampaolesi's line) and as dense, dark pigment accumulated unevenly within the $\mathrm{TM}^{24}$ [Photo 4]. The amount of pigment granules varies considerably and correlates well with the severity of the disease.

Ultrastructural studies indicate that there is active exfoliation production in the TM, Schlemm canal and collector channels as well as passive deposition of PEX material within intertrabecular spaces. The progressive accumulation of the PEX material leads to swelling of the juxtacanalicular meshwork and gradual narrowing and disorganisation of the Schlemm canal architecture in advanced cases. Occasionally, proliferating and migrating corneal endothelial cells produce a pretrabecular sheet of abnormal extracellular matrix that covers the inner surface of the uveal meshwork. ${ }^{25}$

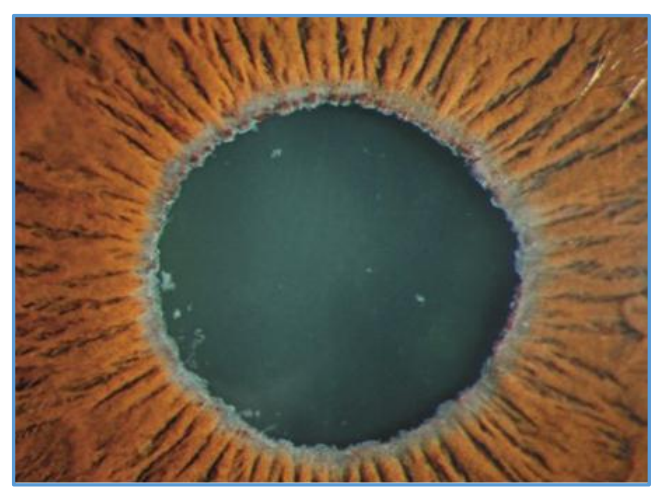

Photo 1. PEX Material on Pupillary Border 


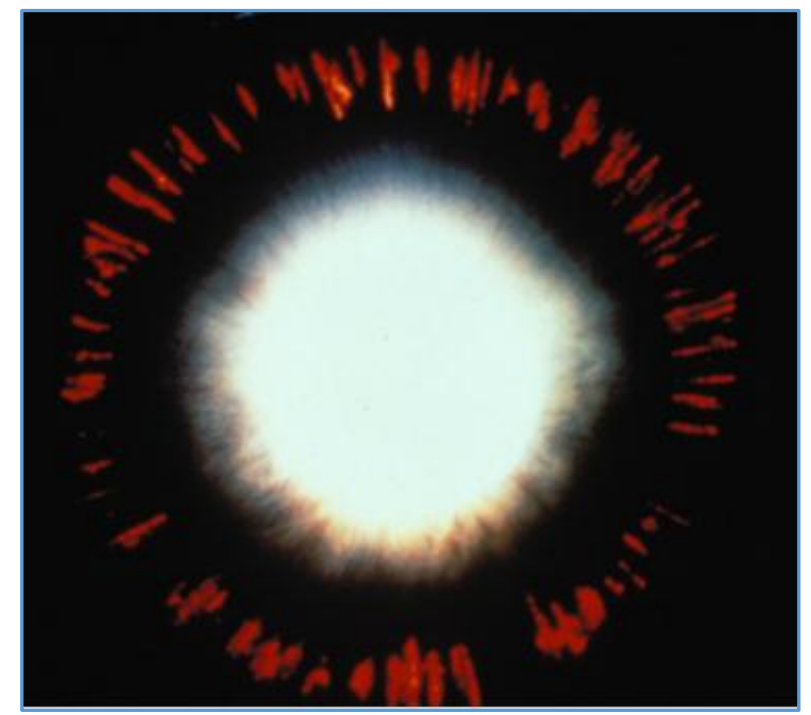

Photo 2. Transillumination Defects

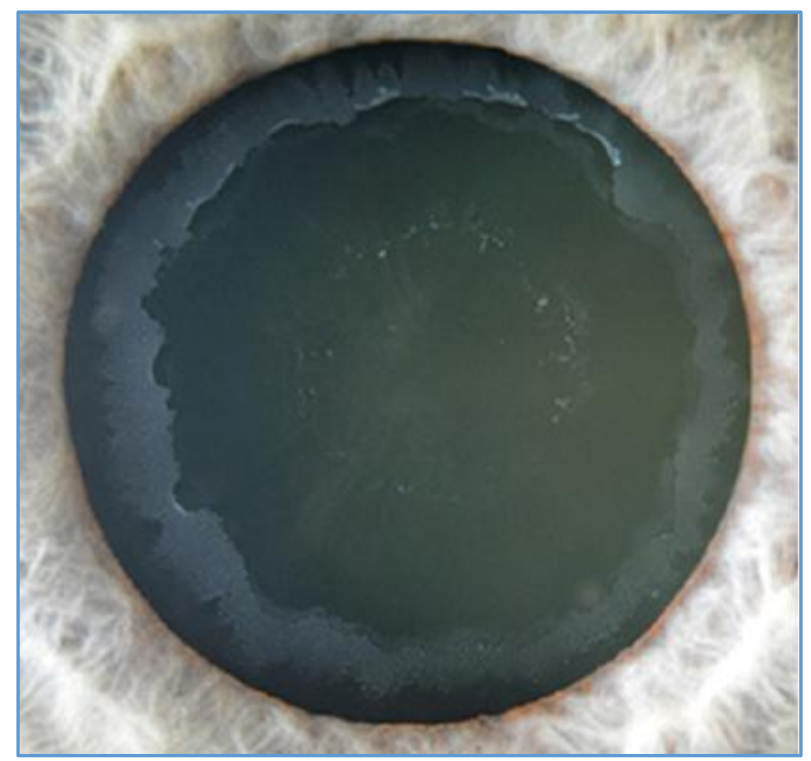

Photo 3. PEX Material on Anterior Lens Capsule

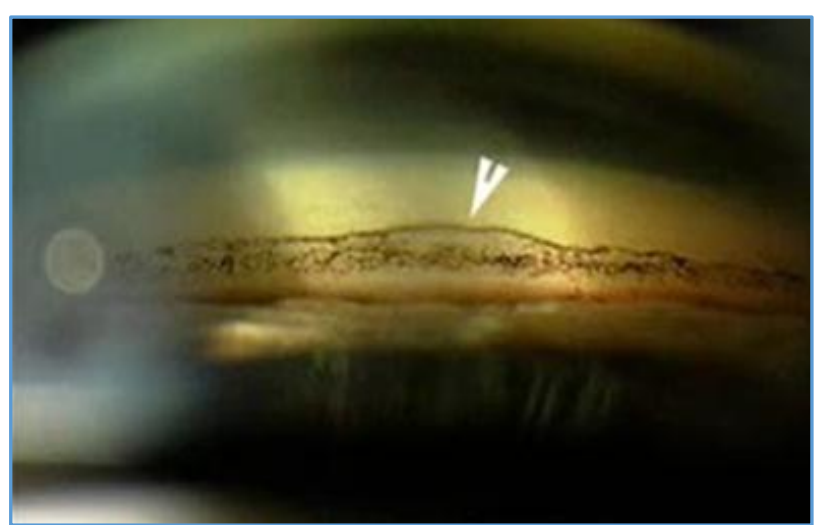

Photo 4. Sampaolesi Line

\section{Aqueous Humour Changes}

Aqueous humour production in PXFS eyes has been demonstrated to be reduced.15 Breakdown of blood aqueous barrier and leakage of serum from iris vessels has been demonstrated to occur in PXFS eyes. ${ }^{26}$ This is associated with consequent presence of higher levels of aqueous protein concentration as well as sudden changes in levels of acid phosphatase, alpha-1 lipoprotein and ceruloplasmin, cellular/plasma fibronectin, transferrin, alpha-1 antitrypsin and growth factors. ${ }^{15}$ There are also increased levels of reactive oxygen species related to increased oxygen content in the aqueous humour of PXFS eyes.

\section{Evolution of PXFG from PXFS}

In a prospective 10 years study, almost one-third of observed patients converted from unilateral to bilateral presence of PEX material in 5 years and almost $40 \%$ in 10 years with a mean conversion time to bilateral PXFS presence of over 5 years. ${ }^{27}$ In a community-based study, similar results were observed with a third of patients converting to bilateral PXFS in 15 years. ${ }^{28}$ In the early manifest glaucoma trial, $55 \%$ of the patients with PXFS developed glaucoma after a mean observation period of 8.7 years. ${ }^{29}$ In other studies, a third of the eyes with PEX material developed glaucoma within 10 years, ${ }^{27}$ while an even higher rate of $44 \%$ required treatment for glaucoma after 15 years of followup. ${ }^{28}$

The risk factors for conversion to PXFG include the presence of PEX material (within ocular tissue and bilateral ocular involvement), ocular hypertension, ${ }^{29}$ decreased pupillary dilatation ${ }^{27}$ and the presence of pigment on the angle structures. ${ }^{30}$ The amount of pigment present in the iridocorneal angle appears to correlate more closely with the severity and development of PXFG than the amount of PEX material on the lens capsule. ${ }^{30}$

It is thought that IOP elevation in PXFS and PXFG is caused by blockage within trabecular meshwork by PEX material and pigment granules released from the posterior iris layer and pupillary margin. It seems likely that the TM becomes overwhelmed with a loss of functional reserve for handling the PEX material and pigment deposits and it may be that the macrophage response of trabecular cells is no longer able to deal with the abnormal material. ${ }^{24}$ Subsequent development of IOP elevation and exaggerated 24 hours IOP fluctuation lead to $\mathrm{ONH}$ damage.

\section{Open-Angle Glaucoma}

Most eyes with PXFG have an open-angle mechanism, although acute angle-closure glaucoma also occurs in a small number of cases.5,31,32 It is also not uncommon for patients with PXFS to have an acute onset of high IOP in the presence of open angles. Once open-angle glaucoma has developed, the IOP tends to run higher and maybe more difficult to control than in cases of POAG. ${ }^{33}$ It has also been observed that the probability of developing glaucomatous optic neuropathy is higher in eyes with PXFG than in other forms of glaucoma at similar IOP levels. ${ }^{32}$ Glaucomatous neuroretinal rim damage tends to be more diffuse with PXFG as opposed to the sectorial preference in POAG. ${ }^{34}$

\section{Angle-Closure Glaucoma}

A less common mechanism of glaucoma in patients with PXFS is acute or chronic angle-closure glaucoma.5,32,35 A number of mechanisms may create a tendency towards pupillary block and angle closure including zonular weakness causing anterior movement of the lens, lens thickening from cataract formation, increased adhesiveness of the iris to the lens due to PEX material, sphincter muscle degeneration, uveitis and iris rigidity from hypoxia. 


\section{Differential Diagnosis}

\section{Capsular Delamination}

Another group of disorders that involve exfoliation of the anterior lens capsule has been referred to as true exfoliation of the lens capsule or capsular delamination. ${ }^{36}$ These cases differ from PXFS in that the underlying precipitating factor such as trauma, exposure to intense heat or severe uveitis is often, but not always present. ${ }^{37}$ The nature of exfoliation also differs with thin, clear membrane-like material separating from the anterior lens capsule and often curling at the margins. ${ }^{38}$ Glaucoma occurs infrequently with capsular delamination.

\section{Primary Amyloidosis}

The amyloid maybe deposited as a white, flaky substance throughout the eye including the pupillary margin of the iris, the anterior lens capsule and the anterior chamber angles creating a clinical picture that resembles PXFS. ${ }^{39}$ In the autosomal dominant condition, familial amyloidotic polyneuropathy, glaucoma is the most common ocular manifestation.

\section{Pigment Dispersion}

Many conditions are characterised by increased pigmentation of the trabecular meshwork. They include the pigment dispersion syndrome and pigmentary glaucoma; some forms of anterior uveitis, melanosis and melanomes; and POAG; and otherwise normal eyes with unusually heavy pigment dispersion.

\section{Medical Management of PXFG}

Medical therapy in PXFG is currently similar to POAG. However, the trend remains for PXFG to respond less well to monotherapy, to require more often adjunctive therapy, maximal medical therapy and finally to require sooner and more often surgical intervention. ${ }^{24}$ In PXFG, response to timolol has been reported to be less favourable than in POAG. ${ }^{40}$ In a 24-hour study, despite a greater mean 24 hours, IOP reduction with timolol, PXFG patients still exhibited worse 24 hours IOP characteristics and greater IOP fluctuation than age-matched patients with POAG. ${ }^{40}$ Dorzolamide was documented to be efficacious as monotherapy in PXFG. ${ }^{41}$ PXFG patients typically have an excellent response to prostaglandin analogues. ${ }^{42}$ The use of miotics has some theoretical advantage for PXFG patients. Miotic agents forcefully open the trabecular meshwork and increase drainage as well as limit pupillary movement. ${ }^{43}$ By reducing pupil motion, less pigment and PEX material are released from their respective location. ${ }^{43}$ In certain PXFG patients with higher baseline IOP and advanced damage, fixed combinations maybe the best initial choice for therapy.

\section{Laser Trabeculoplasty (LTP) in PXFG}

PXFG seems to respond better in the short term than other forms of glaucoma to LTP. Despite the often dramatic IOP lowering achieved with LTP in PXFG, late failures are unfortunately quite common. ${ }^{44}$ This may represent new deposition of PEX material in the outflow pathway. Although, uncertain of the role of repeat treatment with LTP in these late failures, some clinicians apply retreatment.

PXFG is characterised by dense trabecular pigmentation, therefore, PXFG eyes with open anterior chamber angles are particularly suitable for LTP. Since the amount of pigmentation of the meshwork directly influences the outcome and in PXFG the meshwork is generally more heavily pigmented, the power selected should be less than that selected with POAG.

Argon Laser Trabeculoplasty (ALT) has very few complications - the commonest is a transient elevation of IOP, which is more common in PXFG than in POAG. ${ }^{24}$ The laser effect declines overtime and the subsequent failure rate is higher in PXFG (approximately 10\% per year) than that seen in POAG. 45 Despite the poor long-term success, ALT is a valuable treatment option in PXFG, since success of medical therapy and filtration surgery are also worse in PXFG compared with POAG. Primary ALT maybe considered in PXFG. In a long-term study, ${ }^{46}$ the probability of success after primary ALT was $80 \%$ after 2 years, $54 \%$ after 5 years and $36 \%$ after 8 years in PXFG.

Selective Laser Trabeculoplasty (SLT) is a new technique, which selectively targets the intracellular melanin granules in the TM cells. ${ }^{47}$ Using short exposure time, very low power and wide area of laser application, thermal damage and disruption of the TM is reduced. Because of its nondestructive nature, repeated treatments with SLT are possible.

\section{Surgery in PXFG}

Surgery is indicated when medical and laser therapy has failed to obtain the predetermined target pressure or when visual field loss and/or optic disc damage progresses despite conservative treatment.

\section{Filtration Surgery}

Trabeculectomy is still recognised as the standard surgical procedure in progressive uncontrolled PXFG. ${ }^{48}$ Following trabeculectomy, patients with PXFG may progress less frequently than those with POAG. ${ }^{49}$ Trabeculectomy is more successful with the concomitant use of antimetabolites. When PXFG coexists with advanced lens opacities, combined surgery may be considered.

\section{Angle-Based Procedures}

The most commonly performed angle-based procedures are $\mathrm{Ab}$ interno trabeculectomy and trabecular aspiration.

According to Jacobi et al, trabecular aspiration tended to regress after 2 to 4 years of followup because of new accumulation of PEX material.50

Ting et al showed one year results of $\mathrm{Ab}$ interno trabeculectomy, known as Trabectome (NeoMedix Corp., Tustin, CA) surgery in patients with POAG versus PXFG. They demonstrated that IOP reduction and decrease in medication could be obtained in both groups after Trabectome treatment. Moreover, a more significant rate of surgical success was obtained in the PXFG group with $72.1 \%$ versus 62.9\% of success achieved in POAG and PXFG, respectively. 51

Jordan et al found similar IOP reduction in PXFG patients who underwent Trabectome or combined Trabectome and cataract surgery. 52

Carassa et al compared viscocanalostomy against trabeculectomy in 50 patients. After 2 years of follow-up, the success rates (IOP $<21 \mathrm{mmHg}$ and no medication) were $76 \%$ for the viscocanalostomy and $80 \%$ for the trabeculectomy groups. Furthermore, when the IOP target was lowered to 16 $\mathrm{mmHg}$, they obtained a success rate of $56 \%$ and $72 \%$, respectively. ${ }^{53}$ 
The results of viscocanalostomy are encouraging in PXFG patients; however, there is some reluctance regarding this technique, because the final IOP target achieved is still not adequate for patients with advanced glaucoma.

\section{Express Implants (Alcon Laboratories Inc., Fort Worth TX)}

In a recent study conducted by Moisseiev et al, trabeculectomy and the Express technique used and compared in patients with POAG, PXFG and "complex glaucoma" and no differences in terms of surgical success between these procedures were reported. ${ }^{54}$

\section{Aqueous Shunt Implants}

With regard to aqueous shunt implants in eyes with PXFG, there have been fewer studies. The recent "Tube Versus Trabeculectomy (TVT) Study" enrolled a relatively small number of PXFG subjects who had previous ocular surgery. Similar to other studies, no surgical complications unique to PXFG were observed in the TVT study. ${ }^{55,56}$ Similar to trabeculectomy, the surgeon needs to be cautious of postoperative inflammation in PXFG eyes, especially when the tube opens several weeks later in non-valved devices. The period of tube opening is highly inflammatory for reasons that are unclear and the inflammation can be greater in PXFG eyes compared with that observed in patients with POAG.57

\section{Endocyclophotocoagulation (ECP)}

ECP is performed intraocularly with the aid of an endoscope (usually in conjunction with cataract extraction), which permits direct visualisation of the targeted ciliary body tissue. In a case series of 68 patients (including those with PXFG), researchers documented a $34 \%$ mean IOP reduction following ECP. Although, a large prospective study of ECP in eyes with PXFG has not been performed, this seems likely a promising therapy going forward.

\section{Cataract Surgery in PXFS/PXFG}

Although, lens extraction is not advocated for the management of PXFG, cataract extraction for improvement of visual acuity is frequently indicated and requires special considerations in these patients. Patients with PXFS have a higher than average risk of zonular and capsular breaks. ${ }^{58}$ This is most likely caused by degeneration of zonular fibrils, but it may also be associated with a thin posterior lens capsule. ${ }^{59}$

\section{CONCLUSION}

Clinically observed PEX deposits in intraocular surfaces may represent the "tip of an iceberg" of a more fundamental disease process affecting the organisation of elastic fibre networks. These molecular and pathophysiologic events may underlie uncontrolled IOP, glaucoma, lenticulozonular complex weaknesses, surgical complications and higher rates of postoperative inflammation seen in PXFG.

\section{REFERENCES}

[1] Ringvold A. Epidemiology of the pseudo-exfoliation syndrome. A review. Acta Ophthalmol Scand 1999;77(4):371-5.
[2] Ariga M, Nivean M, Utkarsha P. Pseudoexfoliation syndrome. Journal of Current Glaucoma Practice 2013;7(3):118-20.

[3] Astrom S, Stenlund H, Linden C. Incidence and prevalence of pseudoexfoliation and open-angle glaucoma in northern Sweden: II. Results after 21 years of follow-up. Acta Ophthalmol Scand 2007;85(8):832-7.

[4] Ritch R, Schlotzer-Schrehardt U. Exfoliation Syndrome. Surv Ophthalmol 2001;45(4):265-315.

[5] Roth M, Epstein DL. Exfoliation Syndrome. Am J Ophthalmol 1980;89(4):477-81.

[6] Thorleifsson G, Magnusson KP, Sulem P, et al. Common sequence variants in the LOXL 1 gene confer susceptibility to exfoliation glaucoma. Science 2007;317(5843):1397-400.

[7] Sein J, Galor A, Sheth A, et al. Exfoliation syndrome: new genetic and pathophysiologic insights. Curr Opin Ophthalmol 2013;24(2):167-74.

[8] Naumann GO, Schlotzer-Schrehardt U, Kuchle M. Pseudoexfoliation syndrome for the comprehensive ophthalmologist. Intraocular and systemic manifestations. Ophthalmology 1998;105(6):951-68.

[9] Vazquez LE, Lee RK. Genomic and proteomic pathophysiology of pseudoexfoliation glaucoma. Int Ophthalmol Clin 2014;54(4):1-13.

[10] Naumann GOH. Pathologie des Auges. $2^{\text {nd }}$ edn. Vol 2. Berlin: Springer 1997:1264.

[11] Schlotzer-Schrehardt U, Kuchle M, Naumann GOH. Electron-microscopic identification of exfoliation material in extrabulbar tissue. Arch Ophthalmol 1991;109(4):565-70.

[12] Schlotzer-Schrehardt UM, Koca MR, Naumann GOH, et al. Pseudoexfoliation syndrome. Ocular manifestation of a systemic disorder? Arch Ophthalmol 1992;110(12):1752-6.

[13] Mitchell P, Wang JJ, Smith W. Association of pseudoexfoliation syndrome with increased vascular risk. Am J Ophthalmol 1997;124(5):685-7.

[14] Visontai Z, Merisch B, Kollai M, et al. Increase of carotid artery stiffness and decrease of baroreflex sensitivity in exfoliation syndrome and glaucoma. $\mathrm{Br} J$ Ophthalmol 2006;90(5):563-7.

[15] Plateroti P, Plateroti AM, Abdolrahimzadeh S, et al. Pseudoexfoliation syndrome and pseudoexfoliative glaucoma: A review of the literature with updates on surgical management. J of Ophthalmol Article ID 370371, 2015;2015:1-9.

[16] Miyake K, Matsuda M, Inaba M. Corneal endothelial changes in exfoliation syndrome. Am J Ophthalmol 1989;108(1):49-52.

[17] Crista AR. Pseudoexfoliation syndrome and cataract surgery in pseudoexfoliation syndrome. Clinic of Ophthalmology. Timisoara Medical Journal 2014 www.tmj.ro/article.php?art=237564682127339.

[18] Prince AM, Ritch R. Clinical signs of the exfoliation syndrome. Ophthalmology 1986;93(6):808-7.

[19] Brooks AM, Gillies WE. The development of microvascular changes in the iris in exfoliation of the lens capsule. Ophthalmology 1987;94(9):1090-7. 
[20] Asano N, Schlotzer-Schrehardt U, Naumann GOH. A histopathologic study of iris changes in pseudoexfoliation syndrome. Ophthalmology 1995;102(9):1279-90.

[21] Layden WE, Shaffer RN. Exfoliation syndrome. Am J Ophthalmol 1974;78(5):835-41.

[22] Seland JH, Chylack LT. Cataracts in the exfoliation syndrome (Fibrillopathia epitheliocapsularis). Trans Ophthalmol Soc UK 1982;102(Pt 3):375-9.

[23] Futa R, Furuyoshi N. Phakodonesis in capsular glaucoma: a clinical and electron microscopic study. Jpn J Ophthalmol 1989;33(3):311-7.

[24] Exfoilation Syndrome and Glaucoma. Chapter 39. The glaucoma book. Vol 1. Springer 2010:507-16.

[25] Schlotzer-Schrehardt U, Naumann GOH. Trabecular meshwork in pseudoexfoliation syndrome with and without open-angle glaucoma. A morphometric, ultrastructural study. Invest Ophthalmol Vis Sci 1995;36(9):1750-64.

[26] Hardenborg E, Botling-Taube A, Hanrieder J, et al. Protein content in aqueous humour from patients with Pseudoexfoliation (PEX) investigated by capillary LC MALDI-TOF/TOF MS. Proteomics Clinical Application 2009;3(3):299-306.

[27] Puska PM. Unilateral exfoliation syndrome: conversion to bilateral exfoliation and to glaucoma: a prospective 10 year follow-up study. J Glaucoma 2002;11(6):517-24.

[28] Jeng SM, Karger RA, Hodge DO, et al. The risk of glaucoma in pseudoexfoliation syndrome. J Glaucoma 2007;16(1):117-21.

[29] Grodum K, Heijl A, Bengtsson B. Risk of glaucoma in ocular hypertension with and without pseudoexfoliation. Ophthalmology 2005;112(3):38690.

[30] Shuba L, Niolela MT, Rafuse PE. Correlation of capsular pseudoexfoliation material and iridocorneal angle pigment with the severity of pseudoexfoliation glaucoma. J Glaucoma 2007;16(1):94-7.

[31] Brooks AM, Gillies WE. The presentation and prognosis of glaucoma in pseudoexfoliation of the lens capsule. Ophthalmology 1988;95(2):271-6.

[32] Gillies WE, Brooks AM. The presentation of acute glaucoma in exfoliation of the lens capsule. Aust N Z J Ophthalmol 1988;16(2):101-6.

[33] Olivius E, Thorburn W. Prognosis of glaucoma simplex and glaucoma capsulare. A comparative study. Acta Ophthalmol 1978;56(6):921-34.

[34] Tezel G, Tezel TH. The comparative analysis of optic disc damage in exfoliation glaucoma. Acta Ophthalmol 1993;71(6):744-50.

[35] Wishart PK, Spaeth GL, Poryzees EM. Anterior chamber angle in the exfoliation syndrome. $\mathrm{Br} \mathrm{J}$ Ophthalmol 1985;69(2):103-7.

[36] Brodrick JD, Tate GW. Capsular delamination (true exfoliation) of the lens. Report of a case. Arch Ophthalmol 1979;97(9):1693-8.

[37] Elschnig A. Detachment of the zonular lamellae in glass blowers [in German]. Klin Monastsbl Augenheilkd 1922;69:732-4.
[38] Cashwell LF, Holleman IL, Weaver RG, et al. Idiopathic true exfoliation of the lens capsule. Ophthalmology 1989;96(3):348-51.

[39] Tsukahara S, Matsuo T. Secondary glaucoma accompanied with primary familial amyloidosis. Ophthalmologica 1977;175(5):250-62.

[40] Konstas AG, Mantziris DA, Gate EA, et al. Effect of timolol on the diurnal intraocular pressure in exfoliation and primary open-angle glaucoma. Arch Ophthalmol 1997;115(8):975-9.

[41] Heijl A, Strahlman E, Sverrisson T, et al. A comparison of dorzolamide and timolol in patients with pseudoexfoliation and glaucoma or ocular hypertension. Ophthalmology 1997;104(1):137-42.

[42] Konstas AG, Hollo G, Irkec M, et al. Diurnal IOP control with bimatoprost versus latanoprost in exfoliative glaucoma: a crossover, observer-masked, three-center study. Br J Ophthalmol 2007;91(6):757-60.

[43] Susanna R, Weinreb R. Answers in glaucoma. Rio de Janeiro, Brazil: Cultura Medica 2005.

[44] Threlkeld AB, Hertzmark E, Sturm RT, et al. A comparative study of the efficacy of argon laser trabeculoplasty for exfoliation and primary openangle glaucoma. J of Glaucoma (In Press) 1996;5(5):311-6.

[45] Odberg T. Laser therapy of exfoliative glaucoma. In: Hollo G, Konstas AGP. eds. Exfoliation syndrome and exfoliative glaucoma. Savona, Italy: Dogma S.r.l 2008: 149-53.

[46] Odberg T, Sandvik L. The medium and long-term efficacy of primary argon laser trabeculoplasty in avoiding topical medication in open-angle glaucoma. Acta Ophthalmol Scand 1999;77(2):176-81.

[47] Hodge WG, Damji KF, Rock W, et al. Baseline IOP predicts selective laser trabeculoplasty success at 1 year post-treatment: results from a randomized clinical trial. Br J Ophthalmol 2005;89(9):1157-60.

[48] Konstas AGP, Jay JL, Marshall GE, et al. Prevalence, diagnostic features and the response to trabeculectomy in exfoliation glaucoma. Ophthalmology 1993;100(5):619-27.

[49] Popovic V, Sjostrand J. Course of exfoliation and simplex glaucoma after primary trabeculectomy. Br J Ophthalmol 1999;83(3):305-10.

[50] Jacobi PC, Dietlein TS, Krieglstein GK. Bimanual trabecular aspiration in pseudoexfoliation glaucoma: an alternative in non - filtering glaucoma surgery. Ophthalmology 1998;105(5):886-94.

[51] Ting JLM, Damji KF, Stiles MC, et al. Ab interno trabeculectomy: outcomes in exfoliation versus primary open-angle glaucoma. Journal of Cataract and Refractive Surgery 2012;38(2):315-23.

[52] Jordan JF, Wecker T, van Oterendorp C, et al. Trabectome surgery for primary and secondary openangle glaucomas. Graefe's Archive for Clinical and Experimental Ophthalmology 2013;251(12):2753-60.

[53] Carassa RG, Bettin P, Brancato R, et al. viscocanalostomy vs. Trabeculectomy. Ophthalmology 2002;109(3):410-11. 
[54] Moisseiev E, Zunz E, Tzur R, et al. Standard trabeculectomy and Ex-Press miniature glaucoma shunt: a comparative study and literature review. Journal of Glaucoma 2015;24(6):410-6.

[55] Gedde SJ, Herndon LW, Brandt JD, et al. Surgical complications in the Tube versus Trabeculectomy Study during the first year of follow-up. Am J Ophthalmol 2007;143(1):23-31.

[56] Gedde SJ, Schiffman JC, Feuer WJ, et al. Treatment outcomes in the tube versus trabeculectomy study after one year of follow-up. Am J Ophthalmol 2007;143(1):9-22.
[57] Desai MA, Lee RK. The medical and surgical management of pseudoexfoliation glaucoma. Int Ophthalmol Clin 2008;48(4):95-113.

[58] Skuta GL, Parrish RK, Hodapp E, et al. Zonular dialysis during extracapsular cataract extraction in exfoliation syndrome. Arch Ophthalmol 1987;105(5):632-4.

[59] Ruotsalainer J, Tarkkanen A. Capsule thickness of cataractous lenses with and without exfoliation syndrome. Acta Ophthalmol (Copenh) 1987;65(4):444-9. 